\title{
A WEAKLY ANALYTIC LOCALLY CONVEX SPACE WHICH IS NOT $K$-ANALYTIC
}

\author{
JUAN CARLOS FERRANDO
}

\author{
(Received 17 March 2008)
}

\begin{abstract}
It is shown that the dual of the space $C_{p}(I)$ of all real-valued continuous functions on the closed unit interval with the pointwise topology, when equipped with the Mackey topology, is a non $K$-analytic but weakly analytic locally convex space.
\end{abstract}

2000 Mathematics subject classification: 54H05, 46A03, 54C35.

Keywords and phrases: analytic and $K$-analytic space, locally convex space, Borel measure.

\section{Introduction}

A question raised in [2] of whether every weakly analytic locally convex space is analytic is answered in the negative. If $C_{p}(I)$ denotes the linear space of all realvalued continuous functions defined on the closed unit interval $I$ of the real line, provided with the pointwise convergence topology, it is shown that the dual $E$ of $C_{p}(I)$ equipped with the Mackey topology is a weakly analytic locally convex space which is not $K$-analytic. This solution provides an interesting link between descriptive set topology, $C_{p}$-theory and locally convex space theory.

\section{Preliminaries}

If $X$ is a completely regular Hausdorff space the linear space $C(X)$ of the real-valued continuous functions on $X$ equipped with the topology of pointwise convergence is denoted by $C_{p}(X)$. The topological dual of $C_{p}(X)$ is denoted by $L(X)$, whereas $L_{p}(X)$ designs the weak* dual of $C_{p}(X)$. By $C_{c}(X)$ we represent the linear space $C(X)$ equipped with the compact-open topology, whose dual is denoted by $C_{c}(X)^{*}$.

Let us recall that $L(X)$ consists of the linear span of the vectors of the standard copy $\delta(X)$ of $X$ in $C_{p}\left(C_{p}(X)\right)$, that is, each $x \in X$ is depicted in $L(X)$ by the evaluation map

This research has been partially supported by project MTM2005-01182 of the Spanish Ministry of Education and Science, co-financed by the European Community (Feder funds).

(C) 2009 Australian Mathematical Society 0004-9727/2009 \$16.00 
$\delta_{x}$ at $x$, defined by $\delta_{x}(f)=f(x)$ for each $f \in C(X)$. This forces $X$ to be represented in $L(X)$ as an algebraic basis. Indeed, if $\left\{x_{1}, \ldots, x_{n}\right\}$ is a finite subset of $X$ with $\sum_{i=1}^{n} \zeta_{i} \delta_{x_{i}}=\mathbf{0}$, where $\left\{\zeta_{1}, \ldots, \zeta_{n}\right\}$ are real numbers and $\mathbf{0}$ stands for the null linear form on $C(X)$, by choosing $f_{i} \in C(X)$ such that $f_{i}\left(x_{i}\right)=1$ and $f_{i}\left(x_{j}\right)=0$ for $i \neq j$, $1 \leq i, j \leq n$, the equation $\left(\sum_{i=1}^{n} \zeta_{i} \delta_{x_{i}}\right)\left(f_{i}\right)=0$ implies that $\zeta_{i}=0$. Hence $L(X)$ consists of the linear span of the basic vectors $\left\{\delta_{x} \mid x \in X\right\}$ of the standard copy of $X$ in $C_{p}\left(C_{p}(X)\right)$. The mapping $\delta: X \rightarrow L_{p}(X)$ defined by $\delta(x)=\delta_{x}$ is a homeomorphism from $X$ onto the (closed) subset $\delta(X)$ of $L_{p}(X)$.

A Hausdorff topological space $Y$ is said to be analytic if it is a continuous image of the universal Polish space $\mathbb{N}^{\mathbb{N}}$. A Hausdorff topological space $Y$ is said to be $K$-analytic if there exists an upper semicontinuous map $T$ from $\mathbb{N}^{\mathbb{N}}$ into the family $\mathcal{K}(Y)$ of all compact subsets of $Y$, such that $\bigcup\left\{T(\alpha) \mid \alpha \in \mathbb{N}^{\mathbb{N}}\right\}=Y$. Different definitions of $K$-analytic spaces have been shown to be equivalent in the completely regular case [4]. Analytic and $K$-analytic spaces have been studied in [6] under the names of Suslin and $K$-Suslin spaces, respectively. Every analytic space is $K$-analytic and a nonseparable compact space is an example of a $K$-analytic space which is not analytic. It can be easily shown that a compact set $K$ is metrizable if and only if the space $C_{p}(K)$ is analytic. A compact Hausdorff space $K$ is said to be Talagrand compact if $C_{p}(K)$ is $K$-analytic. Weakly analytic and $K$-analytic spaces have been extensively investigated in Banach space theory since Talagrand's seminal paper [5]. For instance, it is well known that a Banach space is weakly $K$-analytic (analytic) if and only if its dual unit ball with the relative weak* topology is Talagrand compact (respectively metrizable).

\section{Main theorem}

In what follows $X$ will be the closed interval $I=[0,1]$ equipped with the relative topology of the real line and the subset $\delta(I)$ of $L(I)$ will be represented by $\Delta$. The Mackey topology on $L(I)$ of the dual pair $\langle L(I), C(I)\rangle$ will be denoted as usual by $\mu(L(I), C(I))$, and the corresponding weak topology, namely the weak* topology of $L(I)$, by $\sigma(L(I), C(I))$. The topology on $\Delta$ induced by $\mu(L(I), C(I))$ will be denoted by $\mu$, whereas $\sigma$ will design the relative topology of $\sigma(L(I), C(I))$ on $\Delta$.

LEMMA 3.1. Each nontrivial convergent sequence of $(\Delta, \sigma)$ does not converge in $(\Delta, \mu)$.

PROOF. Let $\left\{u_{n} \mid n \in \mathbb{N}\right\}$ be a nontrivial convergent sequence of $(\Delta, \sigma)$ and let $u$ be its $\sigma$-limit. Then put $a_{n}=\delta^{-1}\left(u_{n}\right) \in I$ for each $n \in \mathbb{N}$. Since $\left\{a_{n}\right\}$ is a nontrivial convergent sequence of $I$, working with a subsequence if necessary we may assume without loss of generality that $\left\{a_{n}\right\}$ is strictly monotone.

We may suppose for instance (the other case is totally analogous) that $\left\{a_{n}\right\}$ is strictly decreasing with $a_{1}<a_{0}=1$ and $a_{n} \rightarrow a=\delta^{-1}(u)$. Then let us consider a sequence $\left\{f_{n} \mid n \in \mathbb{N}\right\}$ of functions in $C(I)$ satisfying the following conditions: 
(1) $\operatorname{supp} f_{n} \subseteq\left[\left(3 a_{n}+a_{n+1}\right) / 4,\left(3 a_{n}+a_{n-1}\right) / 4\right]$, where supp $f_{n}$ means the support of $f_{n}$;

(2) $0 \leq f_{n} \leq 1$;

(3) $f_{n}\left(a_{n}\right)=1$ for each $n \in \mathbb{N}$.

For example $f_{n}$ can be the function on $I$ taking the value zero in $\left[0,\left(3 a_{n}+a_{n+1}\right) / 4\right] \cup\left[\left(3 a_{n}+a_{n-1}\right) / 4,1\right]$ and whose graph in the band $\left[\left(3 a_{n}\right.\right.$ $\left.\left.+a_{n+1}\right) / 4,\left(3 a_{n}+a_{n-1}\right) / 4\right] \times \mathbb{R}$ of $\mathbb{R}^{2}$ is a triangle with vertices at the points $A_{n}\left(\left(3 a_{n}+a_{n+1}\right) / 4,0\right), B_{n}\left(a_{n}, 1\right)$ and $C_{n}\left(\left(3 a_{n}+a_{n-1}\right) / 4,0\right)$ of the plane. Since supp $f_{i} \cap \operatorname{supp} f_{j}=\emptyset$ if $i \neq j$, the functions $f_{n}$ are disjointly supported. Let us write $A_{n}:=\left[\left(3 a_{n}+a_{n+1}\right) / 4,\left(3 a_{n}+a_{n-1}\right) / 4\right]$ for each $n \in \mathbb{N}$.

As is well known, the topological dual $C_{c}(I)^{*}$ of $C_{c}(I)$ can be identified with the space $r c a(\mathcal{B})$ of regular (countably additive) Borel measures defined on the $\sigma$-algebra $\mathcal{B}$ of all Borel subsets of $I$. Note that if $\mu \in \operatorname{rca}(\mathcal{B})$, since $f_{n}(x) \leq \chi_{A_{n}}(x)$ for $0 \leq x \leq 1$, then, with respect to the dual pair $\left\langle C(I), C_{C}(I)^{*}\right\rangle$, one has

$$
\left|\left\langle f_{n}, \mu\right\rangle\right|=\left|\int_{0}^{1} f_{n} d \mu \leq \int_{0}^{1} \chi_{A_{n}}\right| d|\mu|=|\mu|\left(A_{n}\right)
$$

for every $n \in \mathbb{N}$. Given that $A_{i} \cap A_{j}=\emptyset$ for $i \neq j$ and $\mu$ is countably additive, then $\mu\left(A_{n}\right) \rightarrow 0$ in $\mathbb{R}$. Hence $\left\langle f_{n}, \mu\right\rangle \rightarrow 0$, which shows that $f_{n} \rightarrow 0$ in $C(I)$ under the weak topology of the Banach space $C_{c}(I)$.

If $P$ stands for the $\sigma\left(C(I), C_{c}(I)^{*}\right)$-closure of abx $\left\{0, f_{n}: n \in \mathbb{N}\right\}$, the absolutely convex cover of the weakly compact subset $\left\{0, f_{n}: n \in \mathbb{N}\right\}$ of the Banach space $C_{c}(I)$, Krein's theorem ensures that $P$ is an absolutely convex weakly compact set in $C_{c}(I)$. Since $C_{c}(I)^{*} \supseteq L(I)$, it follows that $P$ is an absolutely convex compact set in $C_{p}(I)$.

On the one hand, the fact that $f_{n} \in P$ for all $n \in \mathbb{N}$ implies that, with respect to the dual pair $\langle C(I), L(I)\rangle$,

$$
\sup \left\{\left|\left\langle f, u_{n}\right\rangle\right|: f \in P\right\} \geq\left|\left\langle u_{n}, f_{n}\right\rangle\right|=f_{n}\left(a_{n}\right)=1
$$

holds for every $n \in \mathbb{N}$. On the other hand, one has

$$
\sup \{|\langle f, u\rangle|: f \in P\}=\sup \{|f(a)|: f \in P\}=0 .
$$

Indeed, since $a \notin \bigcup_{n=1}^{\infty} A_{n}$ then $\left\langle f_{n}, \delta(a)\right\rangle=\left\langle f_{n}, \delta_{a}\right\rangle=f_{n}(a)=0$ for each $n \in \mathbb{N}$, which means that $f(a)=0$ for each $f \in \operatorname{abx}\left\{0, f_{n}: n \in \mathbb{N}\right\}$ and hence for every $f \in P$. From (3.1) and (3.2) it follows that

$$
\sup \left\{\left|\left\langle f, u_{n}-u\right\rangle\right|: f \in P\right\} \geq 1
$$

for every $n \in \mathbb{N}$. Consequently, $u_{n} \nrightarrow u$ in $\Delta$ under $\mu(L(I), C(I))$ whilst $u_{n} \rightarrow u$ in $\Delta$ under $\sigma(L(I), C(I))$.

THEOREM 3.2. The locally convex space $E=(L(I), \mu(L(I), C(I)))$ is weakly analytic but not $K$-analytic. 
PRoOF. Since $I$ and $\mathbb{R}$ are analytic sets and the class of analytic spaces is closed, among other properties, under continuous images, countable products and countable unions of subspaces, then $L_{p}(I)$ is an analytic space as a consequence of [1, Proposition 0.5.13]. So $E$ is weakly analytic. Proceeding by contradiction we assume that $E$ is a $K$-analytic space. Note that since $\Delta$ is closed in $L_{p}(I)$, it is closed in $E$ and consequently $\Delta$ is a $K$-analytic set under the relative topology of $E$.

CLAIM 3.3. There is a completely regular $K$-analytic topology $\tau_{2}$ on I stronger than the relativization $\tau_{1}$ of the usual topology of $\mathbb{R}$ to $I$ such that $\delta$ is an homeomorphism from $\left(I, \tau_{2}\right)$ onto $\Delta$, when considered as a subspace of $E$.

Proof. Since $\mu(L(I), C(I))$ is the strongest locally convex topology on $L(I)$ of the dual pair $\langle L(I), C(I)\rangle$, the topology $\mu$ on $\Delta$ induced by $\mu(L(I), C(I))$ is stronger than the Souslin topology $\sigma$ on $\Delta$ induced by $\sigma(L(I), C(I))$. Now let us consider the homeomorphism $\delta:\left(I, \tau_{1}\right) \rightarrow(\Delta, \sigma)$ and let $\tau_{2}$ be the topology on $I$ consisting of the family $\left\{\delta^{-1}(U): U \in \mu\right\}$ of subsets of $I$. Since $\mu$ is stronger than $\sigma$, if $W$ is a $\tau_{1}$-open subset of $I$ then $\delta(W)$ is a $\sigma$-open subset of $\Delta$ and hence a $\mu$-open set. Hence $W=\delta^{-1}(\delta(W))$ is $\tau_{2}$-open and $\tau_{1} \leq \tau_{2}$.

Clearly, $\delta:\left(I, \tau_{2}\right) \rightarrow(\Delta, \mu)$ is continuous. On the other hand, if $V$ is a $\tau_{2}$-open set of $I$ there is a $\mu$-open set $U$ in $\Delta$ with $V=\delta^{-1}(U)$, so that $\delta(V)=U$. This shows that $\delta:\left(I, \tau_{2}\right) \rightarrow(\Delta, \mu)$ is open. Thus $\delta$ is an homeomorphism from $\left(I, \tau_{2}\right)$ onto $(\Delta, \mu)$.

Claim 3.4. There exists a nonempty perfect set $J$ in I where $\tau_{1}$ coincides with $\tau_{2}$.

Proof. Since $\left(I, \tau_{1}\right)$ is a Baire space and $\left(I, \tau_{2}\right)$ is a regular $K$-analytic space, Nakamura's closed graph theorem [3, Theorem] applied to the identity map $\varphi$ : $\left(I, \tau_{1}\right) \rightarrow\left(I, \tau_{2}\right)$ yields a subset $D$ of $I$ with $I \backslash D$ of the first category in $\left(I, \tau_{1}\right)$ such that $\varphi$ is continuous on $D$; hence $\left.\tau_{1}\right|_{D}=\left.\tau_{2}\right|_{D}$. If $\left\{N_{n}\right\}$ is a sequence of nowhere dense subsets of $\left(I, \tau_{1}\right)$ such that $I \backslash D=\bigcup_{n=1}^{\infty} N_{n}$ and $F_{n}$ denotes the $\tau_{1}$-closure of $N_{n}$ in $I$, then $G=I \backslash \bigcup_{n=1}^{\infty} F_{n}$ is a $\tau_{1}$-dense $G_{\delta}$ in $I$ of the second category (hence uncountable) in $\left(I, \tau_{1}\right)$ with $G \subseteq D$. Since $G$ is an uncountable analytic set, it must contain a nonempty perfect set $J$.

If $J$ is the nonempty perfect subset of $\left(I, \tau_{1}\right)$ determined by Claim 3.4 , let us denote by $K$ the compact subset $\delta(J)$ of $L_{p}(I)$. Given that $J$ is a nonempty perfect subset of the compact space $\left(I, \tau_{1}\right)$, then $|J|=2^{\aleph_{0}}$ and there exists a nontrivial (injective) $\tau_{1}$-convergent sequence $\left\{\zeta_{n}: n \in \mathbb{N}\right\}$ in $I$ contained (together with its limit $a$ ) in $J$. So, if we put $v_{n}:=\delta\left(\zeta_{n}\right)$ for each $n \in \mathbb{N}$, then $\left\{v_{n}\right\}$ is a nontrivial $\sigma$-convergent sequence of $K$, hence of $(\Delta, \sigma)$. But since $\left.\tau_{1}\right|_{J}=\left.\tau_{2}\right|_{J}$ by Claim 3.4, the second statement of Claim 3.3 implies that $\left.\sigma\right|_{K}=\left.\mu\right|_{K}$. So $\left\{v_{n}\right\}$ is a nontrivial convergent sequence in $(\Delta, \sigma)$ which also converges in $(\Delta, \mu)$, contradicting Lemma 3.1. 


\section{Acknowledgements}

I would like to thank Professor J. Kąkol for pointing out to me the question of [2] which has motivated the paper. I also wish to thank Professor M. López Pellicer for helpful conversations on the manuscript.

\section{References}

[1] A. V. Arkhangel'skii, Topological Function Spaces, Mathematics and its Applications, 78 (Kluwer Academic Publishers, Dordrecht, Boston, London, 1992).

[2] J. Ka̧kol, M. López Pellicer and W. Śliwa, Weakly $K$-analytic spaces and the three-space property for analyticity, submitted.

[3] M. Nakamura, 'On quasi-Souslin space and closed graph theorem', Proc. Japan. Acad. 46 (1970).

[4] C. A. Rogers, 'Analytic sets in Hausdorff spaces', Mathematika 11 (1968), 1-8.

[5] M. Talagrand, 'Espaces de Banach faiblement $K$-analytiques', Ann. of Math. 110 (1979), 407-438.

[6] M. Valdivia, Topics in Locally Convex Spaces, North Holland Mathematics Studies, 67 (North Holland, Amsterdam, 1982).

JUAN CARLOS FERRANDO, Centro de Investigación Operativa, Universidad Miguel Hernández, 03202 Elche, Alicante, Spain

e-mail: jc.ferrando@umh.es 